\title{
ON THE DENSEST LATTICE PACKING OF CENTRALLY SYMMETRIC OCTAHEDRA
}

\author{
STEFAN CHALADUS
}

\begin{abstract}
The main purpose of this paper is the calculation of the critical determinant, and therefore the packing constant, for any centrally symmetric octahedron. The results are obtained partially by a numerical computation that is not rigorous. As an application, we prove that the lattice of integer vectors perpendicular to any integer vector $\mathbf{n}=\left[n_{1}, n_{2}, n_{3}, n_{4}\right]\left(0 \leq n_{1} \leq n_{2} \leq n_{3} \leq\right.$ $\left.n_{4}, n_{4}>0\right)$ contains a nonzero vector $\mathbf{m} \in \mathbf{Z}^{4}$, the height $\left(h(\mathbf{m})=\max \left|m_{i}\right|\right)$ of which satisfies
\end{abstract}

(i) $h(\mathbf{m})<\left(\frac{4}{3} h(\mathbf{n})\right)^{1 / 3}$ if $n_{4} \leq-2 n_{1}+n_{2}+n_{3}$,

(ii) $h(\mathbf{m})<\left(\frac{27}{19} h(\mathbf{n})\right)^{1 / 3}$ in any case,

(iii) $h(\mathbf{m}) \leq h(\mathbf{n})^{1 / 3}$ if $n_{4} \geq n_{1}+n_{2}+n_{3}$.

The closing examples show that the above estimations cannot be improved.

\section{INTRODUCTION}

In [3], J. V. Whitworth published results on the densest packing of the sections of the cube

$$
|x| \leq 1, \quad|y| \leq 1, \quad|z| \leq 1, \quad|x+y+z| \leq \tau,
$$

where $0<\tau<3$. For $\tau=1$, it turns out that the convex body (1) is linearly equivalent to the regular octahedron - the case investigated for the first time by H. Minkowski [2], who obtained the general conditions for densest packings of three-dimensional symmetric convex bodies.

The aim of the present paper is the calculation of the critical determinant, and therefore the packing constant, for any centrally symmetric octahedron.

Let $\mathscr{W}$ be the centrally symmetric polyhedron (parallelopiped or octahedron) given by the inequalities

$$
\left|a_{i 1} x+a_{i 2} y+a_{i 3} z\right| \leq 1,
$$

where $a_{i j}(i=1,2,3,4 ; j=1,2,3)$ are real numbers. Let $M_{i}$ be the determinant of the matrix obtained from $A=\left(a_{i j}\right)$ by the omission of the $i$ th row. Without loss of generality we may assume that $0 \leq M_{1} \leq M_{2} \leq M_{3} \leq M_{4}$, $M_{4}>0$. The affine transformation

$$
\varphi:\left\{\begin{array}{l}
a_{11} x+a_{12} y+a_{13} z=X, \\
-a_{21} x-a_{22} y-a_{23} z=Y, \\
a_{31} x+a_{32} y+a_{33} z=Z
\end{array}\right.
$$

Received January 9, 1990; revised June 21, 1990.

1991 Mathematics Subject Classification. Primary 11H31, 05B40, 52C17. 
transforms the polyhedron $\mathscr{W}$ into the polyhedron $\varphi(\mathscr{W})$ :

$$
|X| \leq 1, \quad|Y| \leq 1, \quad|Z| \leq 1, \quad\left|M_{1} X+M_{2} Y+M_{3} Z\right| \leq M_{4} .
$$

Let us observe that $M_{1}+M_{2}+M_{3}>M_{4}$ if and only if $\varphi(\mathscr{W})$ (and thus $\mathscr{W}$ ) is the octahedron.

Since the packing constant is invariant under affine transformations, it follows that we may limit ourselves in the sequel to the following sections of the cube:

$$
|x| \leq 1, \quad|y| \leq 1, \quad|z| \leq 1, \quad|a x+b y+c z| \leq 1,
$$

where $0 \leq a \leq b \leq c \leq 1$. In this way (1) takes the form

$$
|x| \leq 1, \quad|y| \leq 1, \quad|z| \leq 1, \quad \begin{cases}|x+y+z| \leq \tau & \text { for } 1 \leq \tau<3 \\ |\tau x+y+z| \leq 1 & \text { for } 0<\tau \leq 1\end{cases}
$$

Indeed, for $0<\tau \leq 1$ it is sufficient to employ the transformation

$$
\varphi:\left\{\begin{aligned}
-\frac{1}{\tau} x-\frac{1}{\tau} y-\frac{1}{\tau} z & =X \\
x & =Y \\
y & =Z
\end{aligned}\right.
$$

\section{THE MAIN RESULT}

In [3], besides critical determinants, Whitworth gives all critical lattices of the sections (1) of the cube. In every case considered, $0<\tau<\frac{1}{2}, \frac{1}{2} \leq \tau \leq 1$, $1 \leq \tau<3$, there exists at least one critical lattice of Class III $[2,3]$.

In view of the great number of various lattice configurations involved in the discussion, I have considered thoroughly all $\mathbf{K}$-admissible lattices of Class III only, where $\mathbf{K}$ is the section (3) of the cube. Finally, I have found all lattices, (5) and (6), with the smallest determinants. The derivation of this fact extends over many pages.

Afterwards, I have checked by means of computer calculations for Class I and Class II that the lattices (5) and (6) are in fact critical, i.e., there is no K-admissible lattice with a determinant smaller than that of (5) or (6). The calcuations were carried out for all $0 \leq a \leq b \leq c \leq 1, a+b+c>1, a=i / 10$, $b=j / 10, c=k / 10$, where $i, j, k$ are integers, where the coordinates of basis vectors of $\mathbf{K}$-admissible lattices were running through integer multiples of $1 / 30$. This being so, it is highly probable that the following conjucture holds (for lattices of Class III it is in fact a theorem).

Conjecture 1. Let $\Delta$ be the critical determinant of (3), i.e., the determinant of a critical lattice of (3).

(i) If $1 \leq-2 a+b+c$, then

$$
\Delta=\left\{\begin{array}{l}
1 \text { for } b=0 \\
1-(b+c-1)^{2} / 4 b c \text { otherwise, }
\end{array}\right.
$$

and the following three-element systems of vectors determine all the critical lattices of Class III (for $b=0$ we put $(b+c-1) / 2 b=(1+b-c) / 2 b=\frac{1}{2}$ ):

$$
\begin{gathered}
{\left[-1,-\alpha_{2}, \alpha_{3}\right],\left[1, \alpha_{2}-1, \frac{b+c-1}{2 c}-\alpha_{3}\right],} \\
{\left[0, \frac{1+b-c}{2 b}, \frac{1-b+c}{2 c}\right],}
\end{gathered}
$$


where $0 \leq \alpha_{2} \leq 1,0 \leq \alpha_{3} \leq(b+c-1) / 2 c$, and $0 \leq a+b \alpha_{2}-c \alpha_{3} \leq(b+c-1) / 2$;

$$
\begin{gathered}
{\left[-1, \alpha_{2},-\alpha_{3}\right],\left[1, \frac{b+c-1}{2 b}-\alpha_{2}, \alpha_{3}-1\right],} \\
{\left[0, \frac{1+b-c}{2 b}, \frac{1-b+c}{2 c}\right],}
\end{gathered}
$$

where $0 \leq \alpha_{2} \leq(b+c-1) / 2 b, 0 \leq \alpha_{3} \leq 1$, and $0 \leq a-b \alpha_{2}+c \alpha_{3} \leq(b+c-1) / 2$;

$$
[-1,0,0],\left[\alpha_{1},-1, \frac{b+c-1}{2 c}\right],\left[0, \frac{1+b-c}{2 b}, \frac{1-b+c}{2 c}\right],
$$

where $0 \leq \alpha_{1} \leq 1$

$$
[-1,0,0],\left[\alpha_{1}, \frac{b+c-1}{2 b},-1\right],\left[0, \frac{1+b-c}{2 b}, \frac{1-b+c}{2 c}\right],
$$

where $0 \leq \alpha_{1} \leq 1$;

$$
\begin{aligned}
& {[1,0,0],\left[0, \frac{b+c-1}{2 b},-1\right],\left[-1, \frac{1+b-c}{2 b}, \frac{1-b+c}{2 c}\right]} \\
& {[1,0,0],\left[0,-1, \frac{b+c-1}{2 c}\right],\left[-1, \frac{1+b-c}{2 b}, \frac{1-b+c}{2 c}\right] .}
\end{aligned}
$$

(ii) If $-2 a+b+c \leq 1 \leq a+b+c$, then

$$
\Delta=\left\{\begin{array}{l}
1 \text { for } a=0, \\
1-(a+b+c-1)^{3} / 27 a b c \text { otherwise, }
\end{array}\right.
$$

and the following three-element systems of vectors determine all the critical lattices of Class III (for $a=0$ we put $\frac{s}{a}=\frac{1}{3}$ ):

$$
\left[1, \frac{s}{b}, 0\right],\left[0,1, \frac{s}{c}\right],\left[\frac{s}{a}, 0,1\right]
$$

and

(6b)

$$
\left[1,0, \frac{s}{c}\right],\left[\frac{s}{a}, 1,0\right],\left[0, \frac{s}{b}, 1\right]
$$

where $3 s+a+b+c=1$.

Let us observe that in the above conjecture we distinguish two cases, whereas the particular section (1) of the cube requires the consideration of three cases. (This is so because the form (1) is equivalent to (4).)

The volume $V$ of $(3)$ is as follows:

(i) if $1<-a+b+c$, then

$$
V=8-2 \cdot \frac{a^{2}+3(b+c-1)^{2}}{3 b c} ;
$$

(ii) if $-a+b+c \leq 1 \leq a+b+c$, then

$$
V=8-\frac{(a+b+c-1)^{3}}{3 a b c}
$$


Hence, from Conjecture 1 we have

Corollary to Conjecture 1. Let $q$ be the packing constant for (3), i.e., $q=$ $V / 8 \Delta$. Considered as a function $q(a, b, c)$, it is decreasing with respect to $a$. Moreover,

(i) if $1 \leq-2 a+b+c$, then $q \geq \frac{35}{36}$, with equality occurring if $a=\frac{1}{2}$, $b=c=1$;

(ii) if $-2 a+b+c \leq 1$, then $q \geq \frac{18}{19}$ with equality occurring if $a=b=c=1$.

\section{Applications AND EXAMPles}

Let the integer vectors $\mathbf{a}_{j}=\left[a_{1 j}, a_{2 j}, a_{3 j}, a_{4 j}\right](j=1,2,3)$ form a basis of the lattice of integer vectors perpendicular to a nonzero integer vector $\mathbf{n}=$ $\left[n_{1}, n_{2}, n_{3}, n_{4}\right]$. Then there exists an integer vector $m$ such that

$$
\mathbf{m} \cdot \mathbf{n}=0 \quad \text { and } \quad 0<h(\mathbf{m}) \leq \lambda
$$

if and only if the system of inequalities

$$
\left|a_{i 1} x+a_{i 2} y+a_{i 3} z\right| \leq \lambda,
$$

where $i=1,2,3,4$, has an integer solution $\left(x_{0}, y_{0}, z_{0}\right) \neq(0,0,0)$. The affine transformation (2) transforms the above system into

$$
|X| \leq \lambda, \quad|Y| \leq \lambda, \quad|Z| \leq \lambda, \quad\left|n_{1} X+n_{2} Y+n_{3} Z\right| \leq \lambda n_{4}
$$

by virtue of Lemma 3 in [1] (without loss of generality we may assume that $\left.0 \leq n_{1} \leq n_{2} \leq n_{3} \leq n_{4}, n_{4}>0\right)$. Since $\operatorname{det} \varphi=n_{4},(7)$ is satisfied if and only if

$$
\lambda^{3} \geq n_{4} / \Delta
$$

where $\Delta$ is the critical determinant of (3) for $a=n_{1} / n_{4}, b=n_{2} / n_{4}, c=$ $n_{3} / n_{4}$.

Therefore, as a consequence of $\S 2$ and (8) we obtain

Theorem 2. Under the assumption of Conjecture 1, for every integer vector $\mathbf{n}=$ $\left[n_{1}, n_{2}, n_{3}, n_{4}\right]\left(0 \leq n_{1} \leq n_{2} \leq n_{3} \leq n_{4}, n_{4}>0\right)$ there exists a nonzero vector $\mathbf{m} \in \mathbf{Z}^{4}$ such that $\mathbf{m} \cdot \mathbf{n}=0$ and

(i) $h(\mathbf{m})<\sqrt[3]{\frac{4}{3} h(\mathbf{n})}$ if $n_{4} \leq-2 n_{1}+n_{2}+n_{3}$,

(ii) $h(\mathbf{m})<\sqrt[3]{\frac{27}{19} h(\mathbf{n})}$ in any case,

(iii) $h(\mathbf{m}) \leq \sqrt[3]{h(\mathbf{n})}$ if $n_{4} \geq n_{1}+n_{2}+n_{3}$.

The two examples below show that the bounds in Theorem 2 cannot be improved. (For the last bound it is sufficient to assume, e.g., $\mathbf{n}=[1,2,4,8]$.)

Example 1. Let for $t=1,2,3, \ldots$

$$
\mathbf{n}_{t}=\left[n_{1},(2 t+1)\left(3 t^{2}+2 t\right),(2 t+1)\left(3 t^{2}+3 t+1\right),(2 t+1)\left(3 t^{2}+4 t+1\right)\right],
$$

where $n_{1}$ is relatively prime to all factors $(2 t+1),\left(3 t^{2}+2 t\right),\left(3 t^{2}+3 t+1\right)$, and $\left(3 t^{2}+4 t+1\right)$.

A basis of the lattice of integer vectors perpendicular to the vector $\mathbf{n}_{t}$ is the following:

$$
\left[2 t+1,-n_{1}, 2 n_{1},-n_{1}\right],[0, t,-2 t-1, t+1],[0,-2 t-1, t, t] .
$$


For $\mathbf{m}_{t} \neq \mathbf{0}$ and $\mathbf{m}_{t} \cdot \mathbf{n}_{t}=0$ we have $h\left(\mathbf{m}_{t}\right) \geq 2 t+1$, and so

$$
\lim _{t \rightarrow \infty} \frac{\min h\left(\mathbf{m}_{t}\right)}{\sqrt[3]{h\left(\mathbf{n}_{t}\right)}}=\sqrt[3]{\frac{4}{3}}
$$

Example 2. Let for $t=1,2,3 \ldots$

$$
\begin{array}{r}
\mathbf{n}_{t}=\left[152 t^{3}-172 t^{2}+64 t-8,152 t^{3}-152 t^{2}+52 t-6,\right. \\
\left.152 t^{3}-144 t^{2}+46 t-5,152 t^{3}-140 t^{2}+42 t-4\right] .
\end{array}
$$

A basis of the lattice of integer vectors perpendicular to the vector $\mathbf{n}_{t}$ is the following:

$$
\begin{gathered}
{[0,2 t-1,-6 t+2,4 t-1],[2 t-1,-6 t+2,2 t, 2 t-1],} \\
{[4 t-1,2 t, 0,-6 t+2] .}
\end{gathered}
$$

For $\mathbf{m}_{t} \neq \mathbf{0}$ and $\mathbf{m}_{t} \cdot \mathbf{n}_{t}=0$ we have $h\left(\mathbf{m}_{t}\right) \geq 6 t-2$, and so

$$
\lim _{t \rightarrow \infty} \frac{\min h\left(\mathbf{m}_{t}\right)}{\sqrt[3]{h\left(\mathbf{n}_{t}\right)}}=\sqrt[3]{\frac{27}{19}}
$$

These examples allow for immediate calculation of the critical determinants, and consequently the packing constants of (3), in the following two cases:

(i) $b=c=1,0 \leq a \leq \frac{1}{2}$, and

(ii) $a=b=c=1$ (and thus for the regular octahedron).

To this end, it is sufficient to find an admissible lattice of determinant $\frac{3}{4}$ in case (i) and an admissible lattice of determinant $\frac{19}{27}$ in case (ii). Then the assumption of Conjecture 1 is not needed in these cases.

\section{ACKNOWLEDGMENT}

The author is indebted to A. Schinzel for suggesting the problem and for his help in the preparation of this article, and to the referee for substantial improvements.

\section{BIBLIOGRAPHY}

1. S. Chaladus, On a decomposition of integer vectors. III, Discuss. Math. (to appear).

2. H. Minkowski, Gesammelte Abhandlungen, Vol. I, p. 354; Vol. II, pp. 1-42, Teubner, 1911.

3. J. V. Whitworth, On the densest packing of sections of a cube, Ann. Mat. Pura Appl. (14) 27 (1948), 29-37.

Institute of Mathematics, Higher School of Pedagogics, 25-363 Kielce, Poland 